\section{Projection of Long Spark upon the Yellow Spot of the Retina.}

THE entopic vision of the fovea centralis by an instantaneous illumination, especially by blue light, may be well known to ophthalmologists. The following observations, which were made during our physical experiments with long disruptive sparks of a characteristic type, may, however, be of some special interest.

A fine straight spark of several centimetres may be produced between two spherical electrodes of $3-5 \mathrm{~cm}$. diameter which are continuously charged up by means of a Wommelsdorf machine with about $1 \mathrm{~m}$. capacity in parallel, provided that a needle-point leakage is attached to the positive lead at a point not too near the spark gap. With this arrangement the usual irregular bending of spark tracks is effectively avoided, and nearly straight long sparks may be drawn successively at regular intervals. By projecting the magnified image of this spark on a ground-glass screen by means of a proper photographic lens, we obtain a fine luminous line of considerable length and of extremely short duration, which appears at the same position of the screen in regular succession. This constancy of spark track can only be obtained with the use of the needle above mentioned.

It is this type of spark which is specially suited for the observation of the physiological effect here in question. Place a blue filter glass in front of the photographic lens, or before the eye, and look at the image of the spark from a suitable distance. Using a single eyo adapted to darkness for a sufficient time, the part of the luminous line corresponding to the foveal region appears very dark and of saturated blue colour. The dark segment seems to make a singular jerky motion, spreading out a little on both sides. This motion can be seen better when a short segment of the spark is observed, screening off the remaining part by an opaque sheet. On looking at a point on the immediate outside of one end of the luminous streak, luminosity seems to move towards the other end. Some observers seem to perceive also a momentary contracting motion of the dark segment at the beginning or the end of the expanding motion.

Outside the foveal region the luminosity appears to move towards the yellow spot. This may be observed most effectively when the eye is fixed at a point outside one end of the long spark image.

Another fact which may perhaps be of special interest is that when the image of the spark is thrown across the centre of the foveal region we may observe a pair of faint circular haloes on the two sides of the spark track. The interior of the halo is quite dark and the two dark discs touch the spark as well as each other. The screen was covered by a piece of cardboard with a slit slightly wider than the spark image, such that the faint illumination of the general background in the neighbourhood of the spark could be cut off. The halo remained. This is probably brought about by the propagation of excitation along the marginal part of the fovea centralis.

With a yellow filter, the foveal part appears brighter than the external part and bounded by dark fringes.

The experiments may be varied widely by altering the intensity and magnification of spark, the distance of the eye from the screen, the time interval of successive sparks, the time of adaptation of the eye to darkness, etc.

The visual angle of the foveal dark segment was measured and found to be considerably different for two observers.

Institute of Physical and Chemical Research, Tokyo.
The Absorption Spectrum of Selenium Dioxide.

THe fact that selenium dioxide, a white solid, gives a brown vapour has been discussed by Meyer and Langner (Ber., $60 \mathrm{~B}, 285$; 1927), who conclude that the colour must be due to the dioxide and cannot be attributed to selenium liberated by dissociation.

The absorption spectrum of selenium dioxide has been partially described in a brief note by E. J. Evans and G. N. Antonoff (Astrophys. Jour., 34, 277 ; 1911), who, using $0.006 \mathrm{gm}$. of the material in an evacuated tube at temperatures ranging from $360^{\circ}$ to $900^{\circ} \mathrm{C}$., observed absorption band heads from $\lambda \lambda 3930$ to $4470 \mathrm{~A}$. and, at the highest temperature, continuous absorption below $\lambda 4200 \mathrm{~A}$. But absorption in this region will not account for the brown colour of the vapour, which is observed, moreover, at temperatures of the order of $300^{\circ}-400^{\circ} \mathrm{C}$. The spectrum has now been photographed in this laboratory on a Hilger E.I quartz spectrograph and banded structure has been found to extend over a much wider range, namely, $\lambda \lambda 3840$ to $5740 \mathrm{~A}$.

Evans and Antonoff's result may be due to the selenium dioxide vapour in the tube not being saturated, and further to the fact that selenium dioxide dissociates rapidly in vacuo. For this reason, in the present work excess of selenium dioxide was taken and the tube filled with oxygen at a pressure of half an atmosphere at room temperature before sealing off.

The pyrex absorption tube was $1 \frac{1}{2}$ metres long and was heated electrically to temperatures ranging from $250^{\circ}$ to $400^{\circ} \mathrm{C}$. according to the region of the spectrum it was desired to observe. I am indebted to $\mathrm{Mr}$. T. W. Parker, of the Chemistry Department of this College, for a supply of freshly prepared selenium dioxide.

In the near ultra-violet and violet a regular series of diffuse heads is observed, some of which have an appearance of a very complex and fine structure. The diffuseness may thus be due to insufficient resolving power. In the blue-green, green and yellow, the obvious regularity disappears, but there are a number of sharp heads, degraded to the red.

This spectrum is presumably due to selenium dioxide, since saturated selenium vapour at a temperature of the order of $400^{\circ} \mathrm{C}$., in a similar tube, was found to give no absorption in the region in question. Evans and Antonoff obtained selenium absorption bands in this region, but at a higher temperature : at $300^{\circ} \mathrm{C}$., absorption was confined to the region $\lambda \lambda 3200-3700$. Further, when an evacuated absorption tube was used in the present work, the spectrum gradually disappeared owing to the dissociation of the selenium dioxide.

The wave-lengths of the band heads are now being measured, and it is hoped to give fuller details elsewhere.

$$
\text { S. F. Evans. }
$$

Physics Department,

Armstrong College (University of Durham), Newcastle-upon-Tyne.

\section{Dissociation Energy of $\mathbf{Z n}_{2}$ Molecules.}

IT has been shown by me (Zeits.f. Phys., 55, $338 ; 1929$ ) that the dissociation energy of $\mathrm{Hg}_{2}$ molecules is greater than has been supposed. Also, for $\mathrm{Cd}_{2}$ molecules several optical data show that we have to deal with high dissociation energy $(23 \mathrm{kcal} . / \mathrm{mol}$. given by Jablonski). The dissociation energy of $\mathrm{Zn}_{2}$ molecules found by Winans cannot be considered as probable, because Jablonski and Kapuscinski have shown that the considerations of Winans led to erroneus value for the dissociation energy of $\mathrm{Cd}_{2}$ molecules. Therefore it seems interesting to make

No. 3153 , VoL. 125] 